\title{
Synergism between diabetic and radiation retinopathy: case report and review
}

\author{
Martin Viebahn, Michael E Barricks, Mark D Osterloh
}

\begin{abstract}
It is suspected that radiation retinopathy is more likely to develop in an eye with preexisting diabetic retinopathy than in a normal eye. However, there is only one report of this occurring, at a radiation dose of 4500 rads. We present a woman with minimal diabetic retinopathy who had breast carcinoma which was treated with chemotherapy but metastasised to the choroid. Within nine months of external beam radiation ( 3000 rads in fractions of 200 rads) a fulminant retinopathy evolved in that eye, while the non-radiated eye showed no change. The histopathology of radiation and diabetic retinopathy and causes for possible synergism are discussed. As this case report shows, radiation oncologists and ophthalmologists need to be aware of the risk that patients with minimal diabetic retinopathy who have undergone chemotherapy may suffer a dramatic visual loss from radiation therapy despite a radiation dose which is considered adequate, safe, and properly fractionated.
\end{abstract}

Radiation damage in tissues may result from direct parenchymal damage, usually in a rapidly dividing cell system such as intestinal epithelium, or from delayed effects caused by damage to vascular and interstitial support structures. ${ }^{1}$ In the eye, radiation retinopathy is a disease resulting from damage to retinal blood vessels. ${ }^{2-5}$ Gass states that, clinically and angiographically, radiation retinopathy is virtually identical to diabetic retinopathy. ${ }^{6}$

Several authors have noted that patients with diabetes or hypertension may be particularly susceptible to radiation induced vascular damage. ${ }^{1578}$ However, we could find only one case report of this occurring. In this case a

\section{Department of}

Ophthalmology, Pacific

Presbyterian Medical

Center, San Francisco, California

M Viebahn

\section{Department of}

Ophthalmology, Kaiser

Permanente Medical

Center, Oakland,

California

M E Barricks

704 So Webster, Green Bay, Wisconsin

M D Osterloh

Correspondence to: Martin Viebahn, MD, Department of Ophthalmology, Pacific Presbyterian Medical Center, 2340 Clay Street, San Francisco, California 94115, USA.

Accepted for publication 28 March 1991

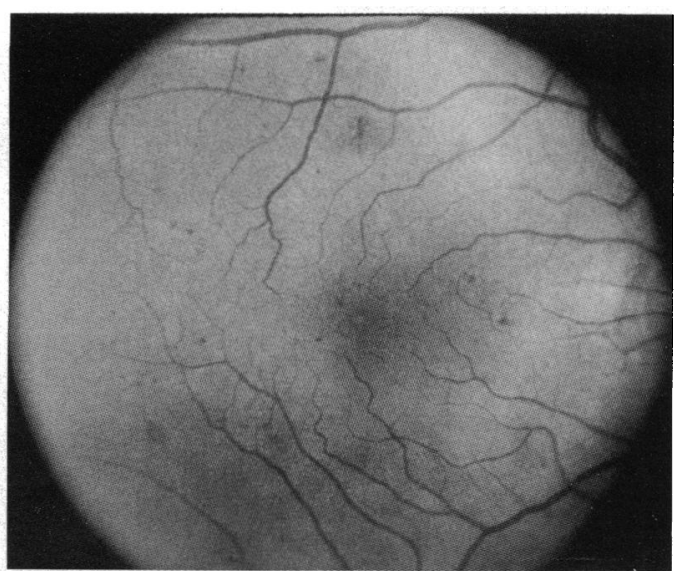

Figure 1 Red-free photograph of the right eye shows minimal background diabetic retinopathy changes with a few microaneurysms and dot haemorrhages. moderate radiation dose ( 4500 rads was given to the fovea by cobalt -60 plaque irradiation) caused radiation retinopathy in a diabetic patient. Chemotherapy is also thought to augment the damaging effects of radiation to the posterior segment of the eye even if it is not concomitant with radiation. ${ }^{157}$

In this paper we describe a case of a woman in which the combined effects of pre-existing minimal diabetic retinopathy, previous chemotherapy, and radiation therapy led to a fulminant retinopathy despite a dose of external beam radiation which was considered adequate, safe, and properly fractionated.

\section{Case report}

The patient is a 54-year-old woman with known insulin dependent diabetes mellitus for 16 years and minimal background diabetic retinopathy. She is otherwise healthy and has no cardiovascular or carotid artery disease. At the age of 45 she underwent a modified radical mastectomy for breast cancer. Since the tumour had spread to adjacent lymph nodes the patient received a full course of cyclophosphamide, methotrexate, and 5-fluorouracil. She developed a cough seven years later, and a mediastinoscopy confirmed the presence of metastatic adenocarcinoma, which has been treated with tamoxifen since.

Nine years after diagnosis of the primary tumour the patient noted photopsias in the right eye. Examination showed a best corrected visual acuity of 20/30 and 20/25 in the right and left eye respectively. There was slight congestion of episcleral vessels of the right eye nasally. Both eyes showed minimal background diabetic retinopathy with a few microaneurysms and dot haemorrhages (Figs 1 and 2). In the right fundus a creamy white choroidal lesion between 3 and 5

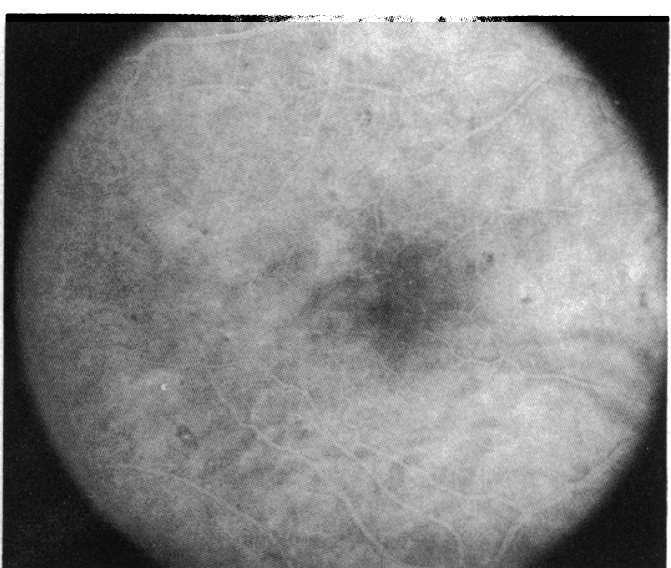

Figure 2 Fluorescein angiogram of the right eye shows microaneurysms and blockage of background choroidal fluorescence by intraretinal haemorrhages (242 seconds after dye injection). 


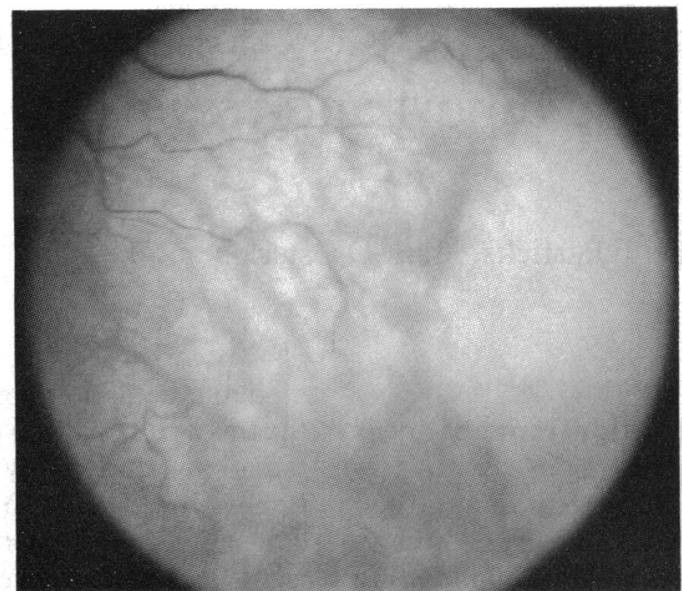

Figure 3 Red-free photograph of the choroidal mass with secondary shallow retinal detachment in the inferonasal periphery of the right eye representing metastatic breast carcinoma.

o'clock in the far inferonasal periphery with secondary shallow retinal detachment was noted, consistent with metastatic carcinoma (Fig 3). A fluorescein angiogram showed very early leakage from this mass lesion which continued throughout the course of the study. A diagnosis of choroidal metastasis from primary breast carcinoma was made. With the $4 \mathrm{MeV}$ linear accelerator the lesion was treated with a total of 3000 rads over three weeks, in fractions of 200 rads.

One month after treatment the secondary retinal detachment had disappeared. The visual acuity and the appearance of mild background diabetic retinopathy were unchanged. Six months after radiotherapy the visual acuity remained unchanged but the retinopathy had markedly increased in the right eye. Cotton-wool spots were now present as well as increased haemorrhages and oedema. Seven months after radiation visual acuity in the right eye had decreased to $20 /$ 80 , and a fluorescein angiogram showed early perifoveal capillary loss as well as haemorrhages and peripheral capillary loss (Figs 4 and 5). Nine months after radiotherapy the vision in the right eye, was hand motions and severe capillary

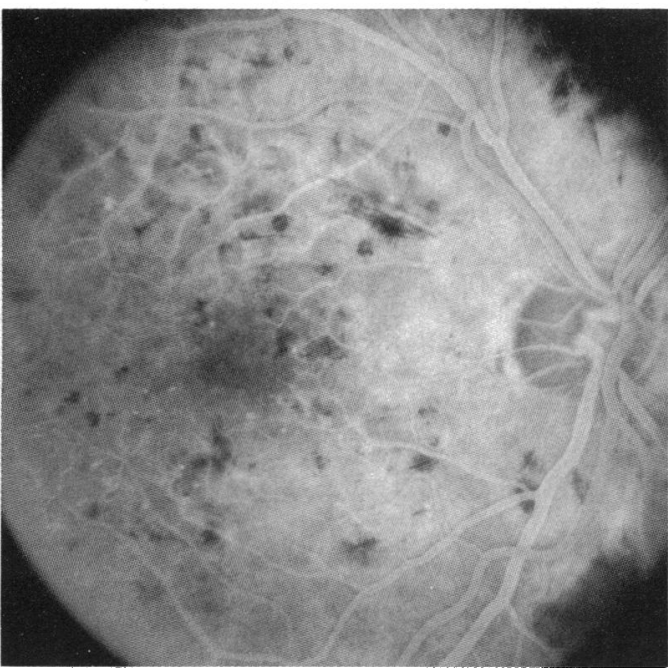

Figure 5 The fluorescein angiogram of the right eye shows early perifoveal capillary loss seven months after radiation therapy (arteriovenous phase).

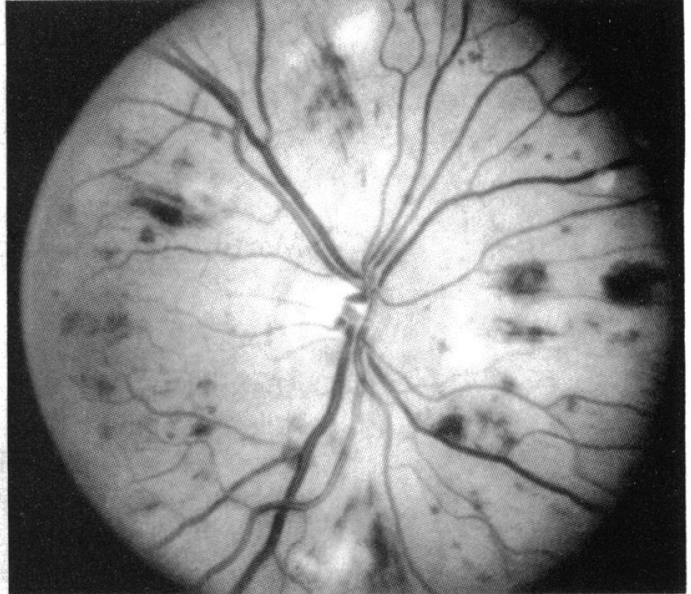

Figure 4 The red-free photograph of the right eye exhibits progression with intraretinal haemorrhages and appearance of cotton-wool spots seven months after radiation therapy.

dropout was seen (Figs 6 and 7). The left eye remained unchanged with $20 / 25$ visual acuity and minimal background diabetic retinopathy (Fig 8).

\section{Discussion}

The first report of radiation retinopathy was published in the 1930s in a case of implantation of radon seeds for the treatment of a retinoblastoma. ${ }^{910}$ Subsequent reports have described the typical clinical and angiographic changes of radiation retinopathy. ${ }^{2-581112}$ Clinically one may see microaneurysms, intraretinal oedema, exudation, and haemorrhage. Retinal telangiectasis, neovascularisation, cotton-wool spots, and vascular sheathing are also seen. Ischaemic optic neuropathy may occur as well as vitreous haemorrhage and retinal detachment. The posterior pole of the eye appears to be the area most sensitive to radiation damage. ${ }^{35}$ On fluorescein angiography the most striking finding is severe retinal capillary non-perfusion. ${ }^{2+6}$ Histologically the walls of small retinal vessels are thickened, and there is loss of endothelial cells. ${ }^{4511} 13$ In experiements on monkeys Irvine and Wood $^{14}$ demonstrated that the retinal capillaries are damaged first. In their trypsin digest flat bed preparations there was initial

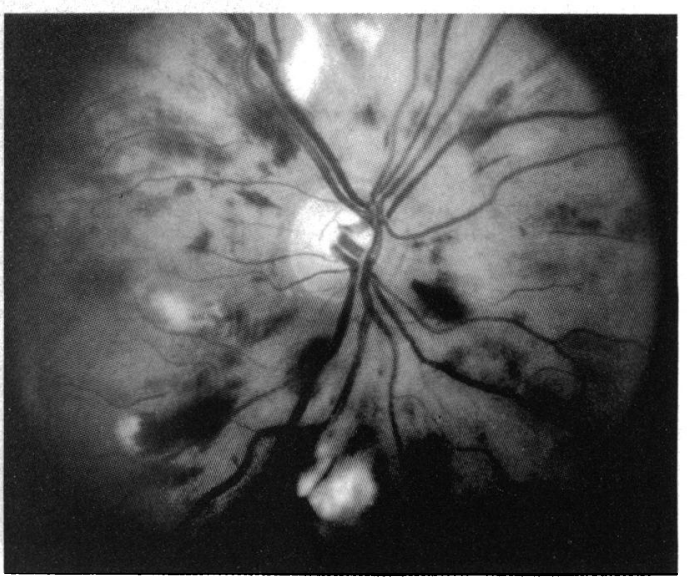

Figure 6 Further progression of the right eye with significan intraretinal haemorrhages and cotton-wool spots nine months after radiation therapy (red-free photograph). 


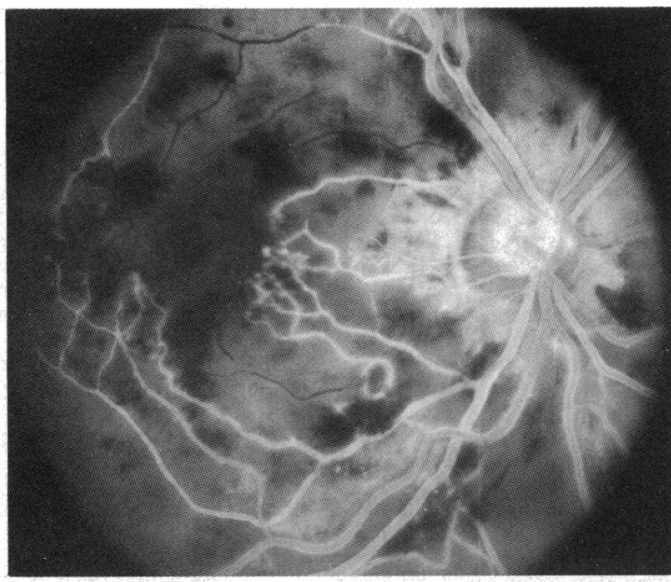

Figure 7 The fluorescein angiogram of the right eye shows marked capillary non-perfusion nine months after radiation therapy (30.6 seconds after dye injection).

endothelial cell loss and then loss of pericytes, resulting in large areas of capillary non-perfusion. Microaneurysms, closure of bigger vessels, recanalisation, and neovascularisation in the retina and to a smaller degree in the choriocapiliaris can be observed later. Electron microscopy confirmed new endothelial cell growth within thickened, recanalised vessels. ${ }^{14}$ However, Irvine $e t a l^{11}$ had found in an earlier study that endothelial cells initially appeared to be less damaged than pericytes on electron microscopy, contrary to the findings in trypsin digest preparations.

Clinically, radiation retinopathy may develop after ionising radiation reaches the posterior segment of the eye by either local treatment (for example, application of a radioactive plaque) or external beam radiation. The radiation may be given for local intraocular lesions (for example, retinoblastoma, choroidal melanoma, metastatic disease) or for adjacent extraocular lesions (for example, nasopharyngeal or sinus carcinoma, or intracranial tumours)..$^{2-5781215}$ The severity of the retinopathy is thought to be related to total radiation dosage and to the fractionation of that dosage. ${ }^{1-3712}$ The total time of the treatment is also of significance.

There is general agreement that 3000 rads with a standard fractionation of 1000 rads per week in five fractions ( 200 rads per treatment session), is a safe total dose of external beam radiation, though there are reports in which radiation retinopathy occurred at lower doses. ${ }^{258}$ However, these dosages were reconstructed, and a low energy $220 \mathrm{KV}$ machine was used which has a higher incidence of complications than contemporary radiotherapeutic emitters. ${ }^{1}$ The time course of retinopathic changes is variable, with an onset between six months and three years after radiotherapy, but earlier and later changes have been noted. ${ }^{5}$ Higher doses of radiation are not known to be associated with a shortened latency period, but the use of adjunct chemotherapy may be. ${ }^{351116}$

Conditions thought to enhance the development of radiation vasculopathy include vascular diseases like diabetes, hypertension, collagen vascular disease, or chemotherapy. ${ }^{157}$ However, we found only one previous report in the literature in which a moderate dose of radiation (4500 rads

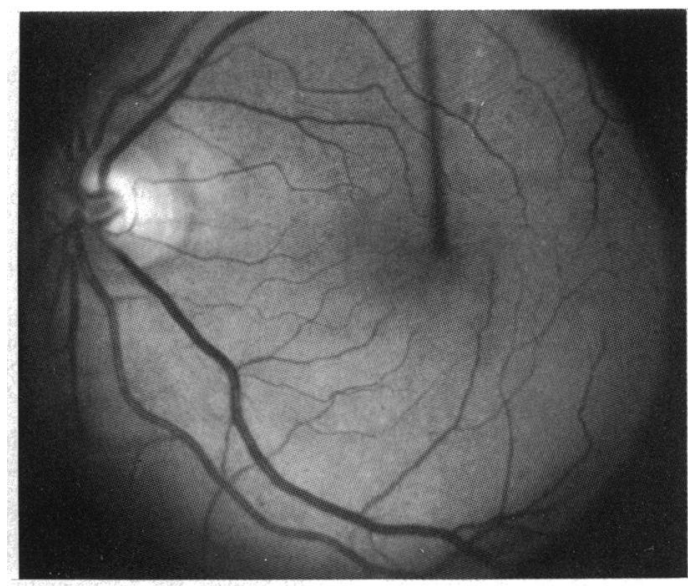

Figure 8 The left eye remains unchanged, exhibiting minimal background diabetic retinopathy changes with a few microaneurysms and dot haemorrhages (red-free photograph).

was given to the fovea by cobalt- 60 plaque irradiation) caused radiation retinopathy in a diabetic patient. ${ }^{5}$ In this study the authors noted that eyes which progressed to total blindness from radiation damage had received chemotherapy more frequently than eyes with less damage. The chemotherapy does not have to be given concurrently with radiation to show this effect. $^{5}$

This case report describes unilateral development of a fulminant retinopathy after orbital radiation and chemotherapy in the setting of minimal background diabetic retinopathy. The patient received 3000 rads in fractions of 200 rads over a three-week period. With our current understanding it is unlikely that in an otherwise normal eye this conservative radiation dose would have led to the described severe and blinding retinopathy. Likewise, the dramatic visual loss was probably not due to mere progression of the pre-existing diabetic retinopathy alone, since the fellow eye was stable throughout the period of observation. This case suggests that pre-existing minimal diabetic retinopathy and prior chemotherapy had a potentiating effect in the pathogenesis of radiation retinopathy. Since both diabetes and radiation primarily cause damage to retinal capillaries, this potentiating effect may not be surprising. In diabetes it has been shown that there is, among other changes such as thickening of basement membrane, an early loss of pericytes. ${ }^{17-20}$ In contrast to this, in radiation induced retinopathy endothelial cells may be disproportionately affected. Since endothelial cells and pericytes are the primary cells making up retinal capillaries, destruction of both these elements by the combined effect of diabetes and radiation would leave little cellular support for capillaries and facilitate changes of capillary closure, aneurysms, vessel leakage, and haemorrhage.

As this case report shows, radiation oncologists and ophthalmologists need to be aware of the risk that patients with minimal diabetic retinopathy who have undergone chemotherapy may suffer a dramatic visual loss from radiation therapy despite a radiation dose which is considered adequate, safe, and properly fractionated.

This study was presented at the West Coast Retina Study Club, 26-27 January, 1987, Kona, Hawaii. 
1 Chako DC. Considerations in the diagnosis of radiation injury. FAMA 1981; 245: 1255-8.

2 Chee PHY. Radiation retinopathy. Am $\mathcal{F}$ Ophthalmol 1968; 66: $860-5$.

3 Bagan SM, Hollenhorst RB. Radiation retinopathy after irradiation of intracranial lesions. Am $\mathcal{F}$ Ophthalmol 1979; 88: 694-7

4 Hayreh SS. Post-radiation retinopathy: a fluorescence fundus angiographic study. Brf Ophthalmol 1970; 54: 705-14.

5 Brown GC, Shields JA, Sanborn G, Augsburger JJ, Savino PJ, Schatz NJ. Radiation retinopathy. Ophthalmology 1982; 89: 1494-501.

6 Gass JDM. Stereoscopic atlas of macular diseases, diagnosis and treatment. 3rd ed. St Louis: Mosby, 1987: 404-5.

7 Wara WM, Irvine AR, Neger RE, Howes EL, Phillips TL. Radiation retinopathy. Int $\mathcal{F}$ Radiat Oncol Biol Phys 1979; 5:

8 Perrers-Taylor M, Brinkley D, Reynolds T. Choroido-retinal damage as a complication of radiotherapy. Acta Radiol Oncol $1965 ; 3: 431-40$.

9 Stallard HB. Radiant energy as (a) a pathogenic (b) a therapeutic agent in ophthalmic disorders. Br $\mathcal{F}$ Ophthalmol 1933 6 (monograph suppl): 69-70.

10 Stallard $\mathrm{HB}$. Glioma retinae treated by radon seeds. $\mathrm{Br} \mathrm{Med} \mathcal{F}$ 1936; i: $962-3$.

11 Irvine AR, Alvarado JA, Wara WM, Morris BW, Wood IS. Radiation retinopathy: an experimental model for the ischemic-proliferative retinopathies. Trans Am Ophthalmol Soc 1981; 79: 103-22.

12 Tomsak RL, Smith JL. Radiation retinopathy in a patient with lung carcinoma metastatic to the brain. Ann Ophthalmol lo80; 12: 619-22.

13 Egbert PR, Fajardo LF, Donaldson SS, Moazed K. Posterior ocular abnormalities after irradiation for retinoblastoma: ocular abnormalities after irradiation for retinoblastoma:

14 Irvine AR, Wood IS. Radiation retinopathy as an experimental Irvine AR, Wood IS. Radiation retinopathy as an experimental
model for ischemic proliferative retinopathy and rubeosis iridis. Am $\mathcal{F}$ Ophthalmol 1987; 103: 790-7.

15 Char DH, Lon LI, Margolis LW. Complications of cobal plaque therapy of choroidal melanomas. Am $\mathcal{F}$ Ophthalmo 1977; 84: 536-41.

16 Chan RC, Shukovsky LJ. Effects of irradiation on the eye. Radiology 1976; 120: 673-5.

17 Kuwahara T, Cogan DG Retinal vascular patterns VI - mural cells of the retinal capillaries. Arch Ophthalmol 1963; 69: cells of the

18 Toussant D, Dustin P. Electron microscopy of normal and diabetic retinal capillaries. Arch Ophthalmol 1963; 70: 96108.

19 Cogan DG, Kuwahara T. The mural cell in perspective. Arch Ophthalmol 1967; 78: 133-9.

20 Speiser P, Gittelsohn AM, Patz A. Studies on diabetic retinopathy III. Influence of diabetes on intramural pericytes. Arch Ophthalmol 1968; 80: 332-7. 